'M Navidinia, 'S Najar Peerayeh, 2F Fallah, 'B Bakhshi, ${ }^{2}$ S Adabian, ${ }^{2} Z$ Gholinejad, ${ }^{2} \mathrm{~S}$ Alimehr, ${ }^{2} \mathrm{MA}$ Malekan. 'Tarbiat Modares University; ${ }^{2}$ Shahid Beheshti University of Medical Sciences, Mofid Children Hospital, PIRC, Tehran, Iran

Background The present study is comparison of commensal and uropathogenic Escherichia coli (UPEC) pathotypes according to multiple virulence genes by multiplex PCR.

Methods Thirty two virulence factors including adhesions group, protectin related genes, common toxins related to UPEC, phylogenic typing, Pathogenicity Island and miscellaneous genes which are involved in Escherichia coli pathogenicity mechanisms were examined by PCR.

Results The PCR assay results identified vat(96 vs. 4\%), PAI IV536(85 vs. $41 \%$ ), PAI ICFT073(78 vs. $25 \%$ ), kpsMTII(71 vs. $42 \%$ ), ompT(68 vs. $53 \%), u s p(52$ vs. $3 \%)$, sat (47 vs. $3 \%)$, picU (41 vs. $1 \%)$, PAI IICFT073(38 vs. $13 \%$ ), PAI I536(37 vs. $7 \%$ ), fliC (H7(24 vs. 9\%)), $h l y D(22$ vs. $2 \%)$, focG(21 vs. $3 \%)$, cnfl(21 vs. $0 \%)$, gafD(20 vs. $2 \%)$ $c d t B$ (19 vs. $7 \%$ ), bmaE(17 vs. $5 \%$ ), PAI II J96(11 vs.3\%), were more frequent virulence markers in urinary E.coli isolates than commensal E.coli, respectively. The frequency of afa(7 vs.10\%), fimH(92 vs. 97\%), kpsMT(K1) (41 vs. 57\%), kpsMTIII(11 vs.5\%), rfc (O4 LPS) ( 9 vs. $2 \%$ ), PAI II536(20 vs. $24 \%$ ), PAI III536(3 vs.0\%), PAI I J96( 53 vs. $43 \%$ ), ibeA(17 vs. $26 \%$ ) markers was almost similar in both of them. cvaC(64 vs. 18\%)was most frequent marker in commensal E.coli. PCR phylotyping revealed higher prevalence of commensal E. coli in groups A and D while uropathogenic strains were mainly found in subgroup B2.

Conclusion Based on these results, the potential for Commensal E.coli to act as human UPEC or as a reservoir of virulence genes for UPEC should be considered.

\section{PHYLOGENIC TYPING OF URINARY E.COLI ISOLATES AND COMMENSAL E.COLI IN PATIENTS WITH UTI IN MOFID CHILDREN HOSPITAL IN IRAN}

doi:10.1136/archdischild-2012-302724.0922

'M Navidinia, 'S Najar Peerayeh, ${ }^{2} \mathrm{~F}$ Fallah, 'B Bakhshi, ${ }^{2} \mathrm{~S}$ Adabian, ${ }^{2} \mathrm{~S}$ Alimehr, ${ }^{2} Z$ Gholinejad, ${ }^{2 M A}$ Malekan. 'Tarbiat Modares University; ${ }^{2}$ Shahid Beheshti University of Medical Sciences, Mofid Children Hospital, PIRC, Tehran, Iran

Background The objectives of this study were to define the phylogenetic groups in urinary and commensal E. coli isolated from urine and stool samples of hospitalized children and to determine the pattern of resistance to antibiotics.

Method A total of 100 urine and stool samples were processed during the study period from September 2009 to August 2010. Samples were cultured using standard microbiological techniques. Biochemical testing was used to identify the organisms, E. coli isolates were tested for phylogenic grouping by using triplex PCR and antibiotic susceptibility test done by the Kirby Bauer method.

Results Phylogenic group B2 and D were predominant in urinary samples (54\% and 34\% respectively). Phylogenic group A, D and B2 were found in decreasing order of $41 \%, 26 \%$ and $16 \%$ respectively in the stool samples.

Following resistance patterns were observed in urinary E. coli isolates vs. commensal $E$. coli, respectively: nitrofurantoin( $2 \%$ vs. $8 \%$ ); imipenem ( $2 \%$ vs. $1 \%$ ); amikacin ( $4 \%$ vs. $3 \%$ ); ciprofloxacin( $8 \%$ vs. $5 \%$ ); nalidixic acid (8\% vs. $27 \%$ ); amoxicillin (16\% vs. $20 \%$ ); ceftazidime (12\% vs.7\%); amoxicillin-clavulanic acid (14\% vs. $9 \%)$; gentamicin $(22 \%$ vs. $12 \%)$; cefpodoxime ( $48 \%$ vs. $19 \%)$; cefotaxime $(45 \%$ vs. $27 \%$ ); co-trimoxazole (61\% vs. $82 \%$ ); and aztreonam (78\% vs. $16 \%)$; Multi-drug resistance (MDR = resistance in $>3$ drugs) was most commonly associated with UPEC isolates.

Conclusion Although group B2 E. coli strains were uncommon in stool samples, as they are highly virulent they still represent a potential reservoir for urinary tract infection. Resistance to most antimicrobials is high both in UPEC and commensal strains.

\section{MULTIPLEX PCR FOR DETECTION OF SHIGA-LIKE TOXIN AND PLASIMID ANTIGEN H GENES OF DIARRHEAGENIC ESCHERICHIA COLI}

doi:10.1136/archdischild-2012-302724.0923

${ }^{1} \mathrm{MM}$ Soltan Dallal, 'Z Rajabi, 'A Akbari, 'S Heidarzadeh, ${ }^{2} \mathrm{MK}$ Sharifi Yazdi. 'Pathobiology, School of Public Health, Tehran University of Medical Sciences; 'Laboratory, School of Paramedical Sciences, Tehran University of Medical Sciences, Tehran, Iran

Background and Aims Despite the fact that Shiga-like toxin E. coli has been identified as a major etiologic agent of children with diarrhea worldwide, few studies have been performed to evaluate the etiology of Shiga-like toxin-producing Escherichia coli (SLTEC) in children with diarrhea, in Iran. The aim of this study was to evaluate the etiology of Shiga-like toxin-producing Escherichia coli (SLTEC) in children with diarrhea, in Iran.

Method A total of 300 stool specimens from children of 300 children with diarrhea were tested for the detection of E. coli, according to standard methods. Out of 300 specimens, 39 were identified as diarrheagenic E. coli, and subjected to multiplex polymerase chain reaction (MPCR) for detection of $\operatorname{st} \times 1 /$ st $x 2$, eae and ipaH genes. We designed a single multiplex polymerase chain reaction (MPCR) for the detection of target genes in diarrheagenic Escherichia coli.

Results EPEC was the dominated strain (55.6\%) among the tested isolates, followed by EHEC (25\%) and EIEC (19.4\%) strains.

Conclusions Shiga-like toxin E. coli has been identified as a major etiologic agent of children with diarrhea in Iran. our method proved to be specific and rapid in detecting virulence genes from Shiga toxin-producing (stx $1, s t \times 2$, and eae), enteropathogenic (eae), enteroinvasive (ipaH) Escherichia coli in stool samples, and able to simultaneously detect of diarrheagenic $E$. coli strains in a single reaction.

\section{DIAGNOSIS AND PROGNOSIS OF CONGENITAL TOXOPLASMOSIS}

doi:10.1136/archdischild-2012-302724.0924

${ }^{1} M G$ Capretti, 'M De Angelis, 'M Spinelli, ' $\mathrm{C}$ Marsico, 'E Tridapalli, ${ }^{2} \mathrm{~A}$ Moroni, ${ }^{2} \mathrm{~A}$ Marangoni, 'L Corvaglia, 'G Faldella. 'Department of Obstetrical, Gynaecological and Paediatric Sciences, Operative Unit of Neonatology; ${ }^{2}$ Department of Haematology, Oncology and Laboratory Medicine, Operative Unit of Microbiology and Virology, St. Orsola-Malpighi University Hospital, University of Bologna, Bologna, Italy

Aims Congenital toxoplasmosis can cause neurological impairment and ocular disease. To describe clinical profile of infants with suspected congenital toxoplasmosis.

Methods Observational study of infants born to mothers with a suspected infection with Toxoplasma gondii during pregnancy between 2002 and 2011.

Serological tests were performed at birth: Toxoplasma specific antibodies IgA, IgM, IgG by Enzyme Immune Assay (EIA), Enzyme Linked Fluorescent Assay (ELFA), Western Blot (WB) tests and WBIgG compared analysis for mother-infant pairs. Infants underwent cranial Ultrasound Scanning, fundoscopy examination, Auditory Brainstem Response, and periodic clinical evaluations.

Results One hundred thirty-one infants Toxoplasma IgG-positive at birth were evaluated; 118/131 (90\%) become IgG-negative at 12 months of life.

Congenital toxoplasmosis was confirmed in 13/131 infants $(9.9 \%)$. Transmitters pregnant women seroconverted in the third trimester (mean 28 \pm 8 weeks).

IgM-ELFA test was positive in 9/13 infants; in 4/13 infants IgM positivity was detected by WB test (negative IgM-EIA/ELFA). Three of 6 infants had a different $\operatorname{IgG}-\mathrm{WB}$ reactivity compared to their mothers.

Six of 13 infected infants (46\%) were symptomatic at birth: $2 / 13$ infants developed chorioretinitis; $4 / 13$ had a pathological neuroimaging (4/4 cerebral calcifications, $1 / 4$ ventriculomegaly). None had 